\title{
Gas Phase Infrared Spectroscopy of Cationic Indane, Acenaphthene, Fluorene, and Fluoranthene
}

\author{
Jos Oomens, ${ }^{*}, \dagger$ Gerard Meijer, ${ }^{\dagger, *}$ and Gert von Helden ${ }^{\dagger}$ \\ FOM Institute for Plasma Physics "Rijnhuizen", P.O. Box 1207, 3430 BE Nieuwegein, The Netherlands, and \\ Department of Molecular and Laser Physics, University of Nijmegen, P.O. Box 9010, \\ 6500GL Nijmegen, The Netherlands
}

Received: March 20, 2001; In Final Form: June 22, 2001

\begin{abstract}
Experimental gas phase infrared spectra are presented for four cationic polyaromatic hydrocarbons (PAHs) incorporating a five-membered ring, indane, acenaphthene, fluorene, and fluoranthene. The ions are stored in a quadrupole ion trap and irradiated with the intense narrow-band infrared light of a free electron laser (FEL). When the wavelength is in resonance with an allowed infrared transition in the ion, multiphoton absorption occurs, resulting in dissociation. Recording the fragment ion yield as a function of FEL wavelength gives a background-free infrared spectrum of the parent ion species. Experimental spectra are found to be in good overall agreement with density functional calculations, except for those for the fluoranthene cation.
\end{abstract}

\section{Introduction}

In the infrared emission spectra of many interstellar and circumstellar objects, strong bands are observed at wavelengths of $3.3,6.2,7.7,8.6,11.3$, and $12.7 \mu \mathrm{m}$. It is widely believed that polyaromatic hydrocarbons (PAHs) are the carriers of those so-called unidentified infrared emission bands (UIRs). ${ }^{1-4}$ This belief relies on the general resemblance of the UIRs and typical $\mathrm{CC}$ and $\mathrm{CH}$ stretching and bending mode frequencies of PAHs. Typical intensities of the UIRs indicate that the PAHs occur predominantly in their ionized form. ${ }^{5-7}$ Absorption of a UV photon and rapid internal conversion populate high vibrational states in the ion, and intramolecular vibrational redistribution (IVR) randomizes the population in the high-density bath of vibrational states. ${ }^{3}$ Due to the extremely low collision rate in interstellar space, the ion releases this internal energy via infrared emission at wavelengths corresponding to the UIRs.

Much effort has recently been devoted to the study of infrared spectra of cationic PAHs, mainly motivated by their likely abundance in the interstellar medium (ISM). These studies include theoretical ${ }^{8,9}$ as well as experimental investigations, the latter based mainly on matrix isolation spectroscopy. ${ }^{10,11}$ In those experiments, the neutral PAH molecules are embedded in cryogenic rare gas matrices and ionization is achieved by irradiation with a Xe lamp. However, ionization efficiencies are low, and the weak ion bands may be overlapped by strong bands due to the residual neutrals. The influence of the matrix on spectral positions and intensities is in general unknown. ${ }^{12} \mathrm{Gas}$ phase experiments suffer from limitations imposed by space charge; i.e., obtainable ion densities are too low for standard infrared absorption techniques to be applied. True laboratory infrared emission spectra of UV-excited neutral PAHs have been reported as well, ${ }^{13-15}$ even in the experimentally challenging wavelength range longward of $4 \mu \mathrm{m}$, where an extremely sensitive infrared spectrometer at liquid He temperatures was employed. ${ }^{16-18}$ However, no such data have thus far been

\footnotetext{
* To whom correspondence should be addressed. Fax: +31 306031204 E-mail: joso@rijnh.nl.

†OM Institute for Plasma Physics "Rijnhuizen".

$\doteqdot$ University of Nijmegen.
}

reported for ionized PAHs. Our group has recently reported experimental gas phase spectra of several small cationic PAHs obtained using the intense and widely tunable infrared radiation of a free electron laser combined with sensitive mass-resolved ion detection. ${ }^{19-22}$

The majority of both theoretical and experimental studies has focused on purely aromatic compounds, and only very recently have some infrared spectra of species that include a fivemembered ring been reported..$^{9,23,24}$ Nevertheless, some PAH molecules with five-membered rings are known to be very stable under the influence of incident UV photons, ${ }^{25}$ which is important in view of their possible abundance in the ISM.

Here we present the infrared spectra of four small PAH cations that include a five-membered ring (indane, acenaphthene, fluorene, and fluoranthene) and compare the spectra to density functional theory calculations. After a brief outline of the experimental apparatus, we first discuss the multiphoton absorption processes by which more than 100 infrared photons can be absorbed by a single molecule, which is required to reach the dissociation threshold of the ions.

\section{Experimental Section}

The experimental apparatus has been described in detail previously. ${ }^{21}$ The technique is based on infrared multiphoton dissociation of ions stored in an ion trap and mass-resolved ion detection, as was previously shown to be possible using powerful $\mathrm{CO}_{2}$ lasers. ${ }^{26,27}$ Here we use a widely tunable free electron laser (FEL) as the source of intense and narrow-band infrared radiation. Briefly, vapor phase molecules effuse from a sample compartment and drift toward the center of a Paul type quadrupole ion $\operatorname{trap}^{28}$ where they are ionized by a $\mathrm{KrF}$ excimer laser and instantaneously trapped. During the UV laser pulse, the amplitude of the rf voltage that is applied to the ring electrode ( $2 \mathrm{~cm}$ inner diameter) of the trap is briefly increased so that UV-induced fragments are ejected from the trap. The resolution of the trap is insufficient to remove fragments that have lost only an $\mathrm{H}_{2}$ unit without affecting the parent ion mass peak. Three milliseconds after the UV laser pulse, the FEL beam, focused to a spot of less than $1 \mathrm{~mm}^{2}$, is fired at the trapped 
ion cloud. This delay is assumed to be sufficient to allow decay of any electronic excitation in the ions that may have been induced by the ionization laser pulse. Typically, two FEL pulses are used to irradiate the sample before an extraction pulse $(-200$ $\mathrm{V}$ ) is applied to the endcap electrode nearest the time-of-flight mass spectrometer equipped with a microchannel plate (MCP) detector. The MCP transients are averaged and recorded using a digital 10-bit oscilloscope (Yokogawa model DL4200) and read into a personal computer. The intensities of the fragment ion peaks are recorded against zero background as a function of FEL wavelength. Fragment yields are obtained by ratioing the fragment peak intensity to the total ion yield. Thus, an infrared spectrum of the parent ion species is obtained. Though it was not possible to completely resolve the $\mathrm{H}_{2}$-loss fragment from the strong parent ion peak, the fragmentation into this channel could be observed by the broadening of the parent ion peak. Monitoring the low-mass flank of this peak then gives the infrared spectrum. All samples are purchased from Aldrich and used without further purification except for indane, which, being a volatile liquid at room temperature, was kept in a small container behind a leak valve and was exposed to one freezepump-thaw cycle.

Experiments were carried out using the free electron laser for infrared experiments (FELIX) at our institute. ${ }^{29}$ The output of FELIX consists of $5 \mu \mathrm{s}$ long macropulses, each with picosecond micropulse substructure, at a $10 \mathrm{~Hz}$ repetition rate. The wavelength is continuously tunable between 5 and $250 \mu \mathrm{m}$, and the bandwidth is nearly transform limited and depends on the micropulse duration of $0.3-5$ ps. In the experiments presented here, the fwhm bandwidth was $\sim 0.6 \%$ of the central frequency. The energy per macropulse amounts to $\sim 60 \mathrm{~mJ}$ in this experiment.

\section{Infrared Multiphoton Dissociation}

Dissociation thresholds for cationic PAHs are typically in the range between 6 and $8 \mathrm{eV}$, which requires at least 60 infrared photons at $10 \mu \mathrm{m}$ to be absorbed in a single ion for fragmentation to occur. Obviously, coherent stepwise multiphoton excitation, where all photons are absorbed in one vibrational ladder $\left(v_{\mathrm{i}}=0 \rightarrow v_{\mathrm{i}}=1 \rightarrow v_{\mathrm{i}}=2 \rightarrow \ldots\right)$, is unrealistic due to the "anharmonicity bottleneck" of the vibrational potential, which would cause the molecule to rapidly run out of resonance with the laser. In contrast, multiphoton absorption likely proceeds via a sequential incoherent absorption process, which is based on rapid IVR in molecules with large vibrational state densities. ${ }^{30-32}$ Absorption of an infrared photon is followed by rapid energy redistribution into a bath of (dark) vibrational states. At room temperature, IVR lifetimes in polyaromatic molecules are typically on the order of $\leq 1 \mathrm{~ns} .{ }^{33,34}$ Although the IVR rate is not directly determined by the density of states $(\rho)$, as was shown by various high-resolution experiments on the stretching modes of medium-sized hydrocarbons $\left(\tau_{\mathrm{IVR}} \sim 100 \mathrm{ps}\right)$, but merely by the presence of low-order coupled states ("doorway" states) and the magnitude of their coupling matrix elements, ${ }^{35-39}$ it defines the statistical limit and thus the degree of irreversibility of vibrational energy diffusion. ${ }^{40}$ In addition, these highresolution experiments have shown that the entire available vibrational phase space is explored by the IVR dynamics. ${ }^{34,37,41,42}$ For the species being investigated here, it appears safe to assume an IVR lifetime of $<1$ ns (i.e., the micropulse spacing of the FEL) so that the absorbing (fundamental) vibrational state is rapidly de-excited, allowing a subsequent photon to be absorbed.

This process is often described in terms of a quasi-continuum of vibrational states occurring at high internal energies. ${ }^{30,32,43}$

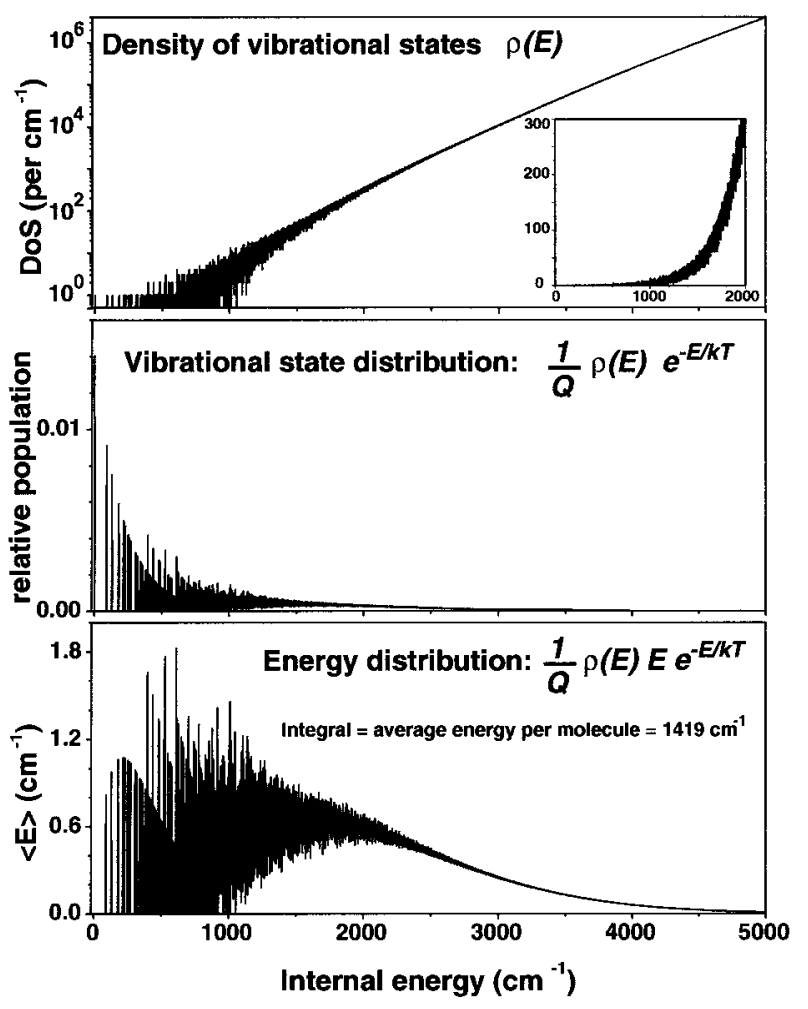

Figure 1. Calculated density of vibrational states for the fluorene cation $\left(\mathrm{C}_{13} \mathrm{H}_{10}{ }^{+}\right)$. The middle panel shows the relative population in each of the states at room temperature, and the bottom panel shows the energy distribution over the vibrational modes. Integration of this distribution yields an average energy per molecule of $1419 \mathrm{~cm}^{-1}$.

Although the name (quasi-)continuum might suggest that photons of any wavelength can be absorbed, this region is characterized by semiresonant absorption in zones near the original fundamental transition frequency. ${ }^{32}$ These zones basically correspond to a convolution of background vibrational states that are (indirectly) coupled to the nearby bright state. The spectral width $(\Gamma)$ of these zones is determined by Fermi's Golden Rule $\left(\Gamma=2 \pi\left\langle W^{2}\right\rangle \rho\right.$, where the mean-squared coupling matrix element $\left\langle W^{2}\right\rangle$ is dominated by the strongly coupled doorway states). ${ }^{37}$ In practice, one observes a spectrum that is slightly red-shifted with respect to the $0 \mathrm{~K}$ spectrum, ${ }^{43}$ due to anharmonic interactions in the bath of vibrational states.

The vibrational state density as a function of internal energy $[\rho(E)]$ was evaluated for the fluorene cation $\left(\mathrm{C}_{13} \mathrm{H}_{10}{ }^{+}\right)$applying the Beyer-Swinehart algorithm ${ }^{44}$ with a grain size of $1 \mathrm{~cm}^{-1}$ and DFT-calculated mode energies (see section 4.3). All vibrational modes were approximated as being harmonic; a graphical representation is shown in the top panel of Figure 1. The 63 normal modes of the ion give rise to a fairly high density of vibrational states already at moderate internal energies.

The room-temperature population distribution $1 / Q \rho(E) \mathrm{e}^{-E / k T}$, where $Q$ is the partition function $\int_{0}^{\infty} \rho(E) \mathrm{e}^{-E / k T} \mathrm{~d} E$, is plotted in the middle panel of Figure 1; it shows for instance that the probability of finding the molecule in its vibrational ground state is only $1.4 \%$. The average energy per molecule is easily obtained by integrating the energy distribution (see the bottom panel of Figure 1)

$$
\langle E\rangle=\frac{1}{Q} \int_{0}^{\infty} \rho(E) E \mathrm{e}^{-E / k T} \mathrm{~d} E=1419 \mathrm{~cm}^{-1}
$$

Thus, at room temperature, the ion already has an internal energy of $1419 \mathrm{~cm}^{-1}$, where the density of states is $\sim 30$ states $/ \mathrm{cm}^{-1}$. 
If an IVR lifetime of $\leq 1 \mathrm{~ns}$ is assumed, ${ }^{33}$ this is near the onset of the quasi-continuum according to the criterion of stochasticity, ${ }^{32}$ which states that the IVR lifetime broadening should exceed the average mode spacing

$$
\frac{1}{2 \pi c \tau_{\mathrm{IVR}}} \geq \rho^{-1}
$$

We therefore conclude that, in the multiphoton process, (most of) the photons are absorbed while the ion resides in the quasicontinuum of vibrational states. Nonetheless, well-resolved spectra are observed (see section 4), in agreement with the existence of narrow resonant sectors in the quasi-continuum. ${ }^{32}$ Note that the internal energy of an ion after the absorption of a UV-visible photon and internal conversion, as proposed in the UIR-PAH hypothesis, ${ }^{3,45}$ is such that subsequent infrared emission occurs while the ion finds itself in the vibrational quasicontinuum as well.

Comparing the typical IVR lifetimes and the length of the FEL macropulse $(5 \mu \mathrm{s})$, one realizes that, if the absorption cross section and laser intensity are sufficient, many cycles of photon absorption and relaxation into the vibrational bath can occur. Thus, it is possible to absorb more than 100 photons, required to reach the dissociation threshold.

In view of this high number of photons required for dissociation, one may wonder how the relative intensities of bands in our multiphoton dissociation spectrum are related to the relative intensities of a linear absorption spectrum. In a previous publication, ${ }^{21}$ we compared our naphthalene cation spectrum to a one-photon linear gas phase absorption spectrum recorded by Piest et al. ${ }^{19}$ It appeared that band intensities in the multiphoton spectrum are very similar to those in the linear absorption spectrum, which is another indication for the sequential photon absorption mechanism as described above. Thus, we shall assume that nonlinearities are negligible and apply a linear laser power correction to all spectra.

Photodissociation dynamics of individual species may vary vastly as is shown by the work of Ekern et al. ${ }^{25}$ Of particular interest in this work is the difference between species losing $\mathrm{C}_{n} \mathrm{H}_{m}$ units and species losing hydrogen only. In Figure 2, timeof-flight transients for indane, acenaphthene, fluorene, and fluoranthene with the FEL on and off a resonance are shown. Off-resonance transients are virtually identical to those taken without the FEL (not shown). The indane and fluorene cations are seen to lose $\mathrm{C}_{2 n} \mathrm{H}_{m}$ units, whereas for the acenaphthene cation, only hydrogen loss is observed. For the fluoranthene cation, carbon-loss channels are very weak, whereas hydrogen loss is observed in the low-mass flank of the parent ion peak. When the TOF traces of the four species are compared, it is not surprising that ions undergoing carbon loss can usually be detected more efficiently than those losing only hydrogen. Modulation of the rf amplitude to remove fragment ions induced by the ionization UV laser, and thus obtaining a flat baseline (see the Experimental Section), is possible in the case of carbon loss only. The mass resolution of the trap is insufficient to remove any background in the $\mathrm{H}_{2}$-loss channel without affecting the parent ion signal, which generally decreases the signal-tonoise ratio for species exhibiting $\mathrm{H}_{2}$ loss only. Due to its relatively low mass, the indane cation forms an exception; most of the background in the $\mathrm{H}_{2}$-loss channel can be removed as is seen in the top left panel of Figure 2.

\section{Infrared Spectra}

4.1. Indane Cation, $\mathrm{C}_{9} \mathrm{H}_{10}{ }^{+}$. Indane may be considered the simplest member of the group of compounds considered in this
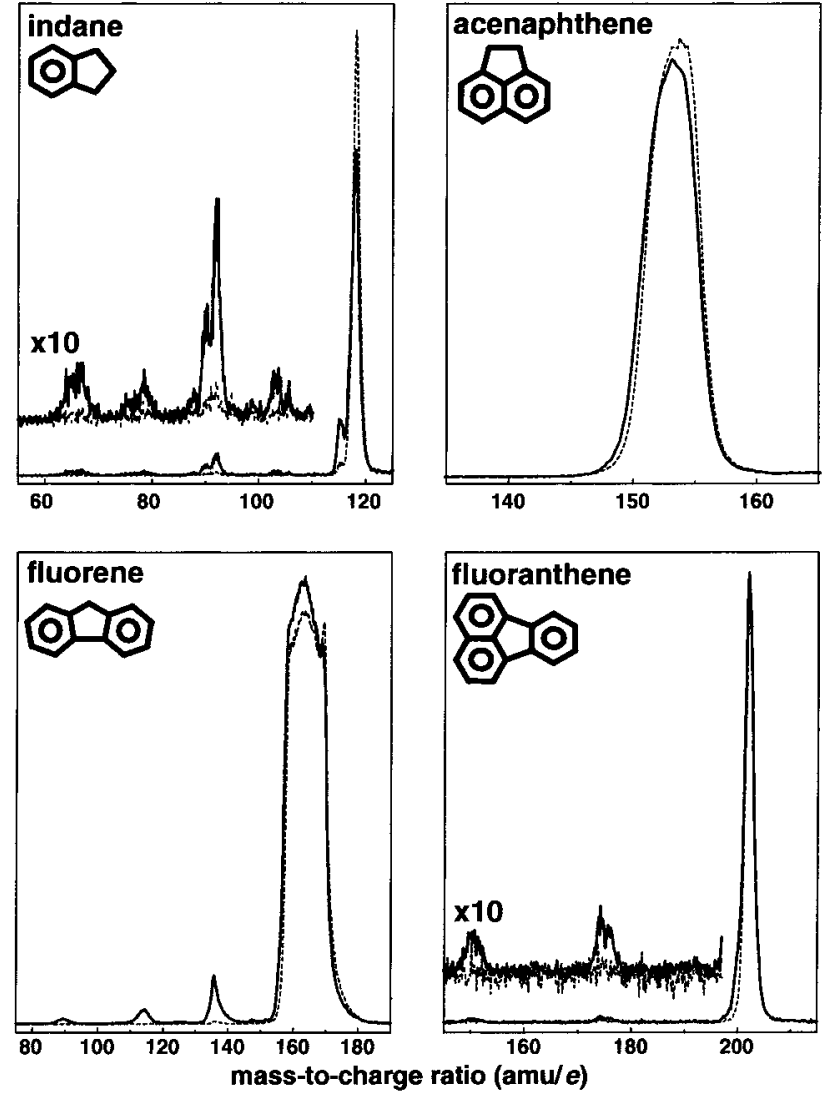

Figure 2. Time-of-flight spectra of the indane, acenaphthene, fluorene, and fluoranthene cations recorded with FELIX on an infrared resonance (solid line) and off resonance (dashed line).

work as it consists of one aromatic ring linked to one fivemembered aliphatic ring. The fragmentation pattern with the FEL tuned in resonance is shown in Figure 2 and shows both $\mathrm{H}_{2}$ loss and a sequence of $\mathrm{C}_{n} \mathrm{H}_{m}$ fragments.

The infrared spectrum was recorded on different fragment channels as shown in Figure 3. The top trace shows the spectrum as recorded on the $\mathrm{H}_{2}$-loss channel. The spectrum below, recorded on the $\mathrm{C}_{2} \mathrm{H}_{2}$-loss channel, shows peaks at the same positions and with the same relative intensities. Spectra recorded on lower-mass fragments (third trace) are different in that several peaks are missing. We first consider the spectrum on the $\mathrm{H}_{2} /$ $\mathrm{C}_{2} \mathrm{H}_{2}$-loss channel, which is rather typical of that of cationic PAHs with strong and partly overlapping features in the 1400 $\mathrm{cm}^{-1}$ range and weaker, well-resolved bands in the out-of-plane $\mathrm{CH}$ bending region at $<900 \mathrm{~cm}^{-1}$. Since, to the best of our knowledge, no infrared spectra of the indane cation, either experimental or theoretical, have been reported to date, we calculated the infrared spectrum using the Becke3LYP density functional ${ }^{46}$ and Dunning's D95(d,p) basis set ${ }^{47}$ as implemented in GAUSSIAN98 (48). A calculation constrained to a planar $C_{2 v}$ geometry yielded one imaginary frequency $\left(-121 \mathrm{~cm}^{-1}\right)$, which appeared to be due to an out-of-plane motion of the outermost carbon atom on the pentagonal ring. Indeed, when this atom is placed slightly out of the skeletal plane and the geometry is reoptimized, convergence to a geometry where the outermost $\mathrm{C}$ atom is $\sim 0.36 \AA$ above the plane is achieved. This $C_{s}$ geometry yields calculated frequencies which are in slightly better agreement with the experimental spectrum than the $C_{2 v}$ geometry. In particular, the bands calculated at 730 and 1192 $\mathrm{cm}^{-1}$ and clearly observable in the experimental spectrum were absent (or very weak) in the calculation based on a $C_{2 v}$ planar carbon skeleton. The $C_{s}$-calculated spectrum, convoluted with 


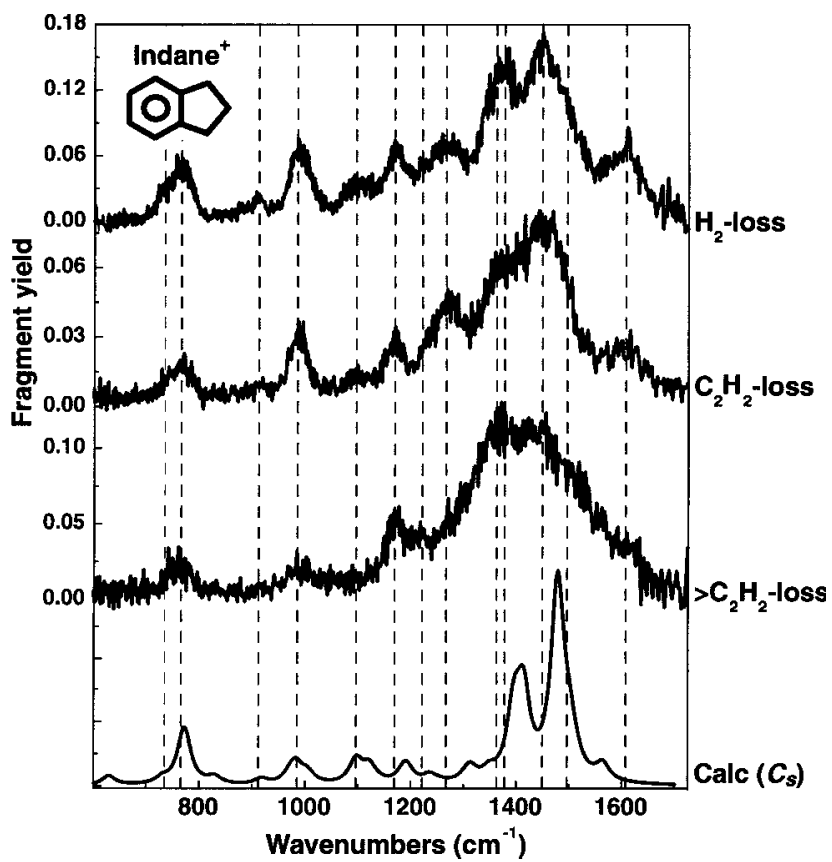

Figure 3. Spectra of the indane cation recorded on different fragment ion mass gates. The top two traces are obtained on the $\mathrm{H}_{2^{-}}$and $\mathrm{C}_{2} \mathrm{H}_{2^{-}}$ loss mass channels. The third trace gives the sum over all other (smaller) fragments. Vertical lines indicate line positions in the $\mathrm{H}_{2}$-loss spectrum as listed in Table 1 . The bottom trace shows the $C_{s}$-calculated spectrum.

TABLE 1: Infrared Spectrum of the indane Cation

\begin{tabular}{|c|c|c|c|c|c|}
\hline \multicolumn{3}{|c|}{ calculated } & \multicolumn{2}{|c|}{ experimental } & \multirow[b]{2}{*}{ approximate description } \\
\hline$C_{s}$ & $v_{\mathrm{vib}}$ & $I_{\mathrm{rel}}{ }^{a}$ & $\nu_{\mathrm{vib}}$ & $I_{\text {rel }}$ & \\
\hline$A^{\prime}$ & 730 & 0.03 & 737 & 0.17 & sym breathing \\
\hline$A^{\prime}$ & 772 & 0.31 & 767 & 0.30 & $\mathrm{CH}$ oop bend \\
\hline$A^{\prime}$ & 829 & 0.04 & & & $\mathrm{CH}_{2}$ perp bend \\
\hline$A^{\prime}$ & 918 & 0.03 & 909 & 0.12 & $\mathrm{CH}_{2}$ bend \\
\hline$A^{\prime}$ & 972 & 0.04 & & & $\mathrm{CH}_{2}$ perp bend/CH oop bend \\
\hline$A^{\prime}$ & 983 & 0.10 & 990 & 0.42 & 6 -ring breathing \\
\hline$A^{\prime}$ & 1004 & 0.05 & & & $\mathrm{CH}_{2}$ wag/ $\mathrm{CH}$ oop bend \\
\hline$A^{\prime \prime}$ & 1097 & 0.13 & 1099 & 0.21 & $\mathrm{CH}$ ip bend \\
\hline$A^{\prime \prime}$ & 1122 & 0.09 & & & $\mathrm{CH}$ ip bend \\
\hline$A^{\prime}$ & 1186 & 0.03 & & & $\mathrm{CH}$ ip bend \\
\hline$A^{\prime}$ & 1192 & 0.08 & 1172 & 0.43 & $\mathrm{CH}$ ip bend \\
\hline$A^{\prime}$ & 1236 & 0.04 & 1225 & 0.26 & $\mathrm{CH}$ ip bend/CC stretch \\
\hline$A^{\prime}$ & 1308 & 0.03 & 1266 & 0.42 & $\mathrm{CH}_{2}$ bend \\
\hline$A^{\prime}$ & 1314 & 0.06 & & & $\mathrm{CH}_{2}$ bend \\
\hline$A^{\prime \prime}$ & 1349 & 0.05 & & & $\mathrm{CH}$ ip bend \\
\hline$A^{\prime \prime}$ & 1396 & 0.32 & 1360 & 0.62 & $\mathrm{CH}_{2}$ asym scissor \\
\hline$A^{\prime}$ & 1397 & 0.04 & & & $\mathrm{CH}$ ip bend/CC stretch \\
\hline$A^{\prime}$ & 1414 & 0.43 & 1383 & 0.80 & $\mathrm{CH}_{2}$ sym scissor \\
\hline$A^{\prime \prime}$ & 1473 & 0.07 & & & $\mathrm{CC}$ stretch/CH ip bend \\
\hline$A^{\prime}$ & 1479 & 1.00 & 1447 & 1.00 & $\mathrm{CC}$ stretch/CH ip bend \\
\hline$A^{\prime}$ & 1502 & 0.20 & & & $\mathrm{CH}_{2}$ scissor \\
\hline$A^{\prime}$ & 1564 & 0.09 & 1602 & 0.39 & $\mathrm{CC}$ stretch \\
\hline
\end{tabular}

${ }^{a}$ Only bands with an $I_{\text {rel }}$ of $\geq 0.03$ are listed.

a $30 \mathrm{~cm}^{-1}$ fwhm Lorentzian line profile, is shown as the bottom trace in Figure 3. As seen in Table 1, the low symmetry yields a wealth of infrared allowed bands, though many of them are weak or unresolved.

Comparing theoretical and experimental spectra, one notices that the $C_{s}$ geometry yields an acceptable agreement. Except for the bands observed at 1099 and $1602 \mathrm{~cm}^{-1}$, all bands are slightly red-shifted with respect to the calculations as one might expect due to anharmonicities involved in the multiphoton absorption process. ${ }^{21,32,49}$ Note also that calculated frequencies were not scaled which generally leads to a slight overestimation. Blue shifts of the calculated frequencies are typically on the order of $1-3 \%$, and experimental line widths are $\sim 30 \mathrm{~cm}^{-1}$ as was previously found for various small PAHs. ${ }^{21}$

The relative intensity of the band near $990 \mathrm{~cm}^{-1}$ appears to be underestimated by a factor of $\sim 4$ in the calculation. In addition, the various weak bands calculated between 1100 and $1350 \mathrm{~cm}^{-1}$ appear to be too weak to explain all the intensity observed in this region. Especially the broad feature near 1266 $\mathrm{cm}^{-1}$ is not well-reproduced in the calculation, and it is tentatively assigned to the parallel $\mathrm{CH}_{2}$ bending modes calculated at 1308 and $1314 \mathrm{~cm}^{-1}$. According to the calculations, the two main features observed near 1375 and $1450 \mathrm{~cm}^{-1}$ consist of partly unresolved bands due to $\mathrm{CH}_{2}$ scissoring and in-plane $\mathrm{CH}$ bending modes. Finally, the calculation fails to correctly reproduce the $\mathrm{CC}$ stretching mode near $1602 \mathrm{~cm}^{-1}$, in both position and intensity.

It is interesting to compare the spectra recorded on different fragment ion mass channels (see Figure 3). The spectrum recorded on the $\mathrm{C}_{2} \mathrm{H}_{2}$-loss channel, shown as the second trace in Figure 3, is very similar to the $\mathrm{H}_{2}$-loss spectrum given by the top trace. The intensities in lower-mass fragment channels (more than $\mathrm{C}_{2} \mathrm{H}_{2}$ loss) are significantly lower as seen in the top left panel of Figure 2. The wavelength spectra recorded on these channels are, however, very similar, and adding them up gives the third trace in Figure 3. This trace is clearly different from the spectrum recorded on the $\mathrm{H}_{2}$ and $\mathrm{C}_{2} \mathrm{H}_{2}$-loss channels. Note that all spectra are recorded simultaneously in one experimental run by sampling different channels of the digital oscilloscope. In the spectrum recorded on the lighter fragments, the bands at 737, 909, 1099, 1266, and $1602 \mathrm{~cm}^{-1}$ have disappeared. In addition, the fairly strong band at $990 \mathrm{~cm}^{-1}$ has become very weak.

The question that now arises is why the spectra for the loss of larger units are different from that for $\mathrm{H}_{2}$ and $\mathrm{C}_{2} \mathrm{H}_{2}$ loss. As a possible explanation, one could think that different normal modes couple differently to the various fragmentation channels. However, to reach the dissociation threshold, at least 50-100 infrared photons need to be absorbed, and at such a level of vibrational excitation, the energy distribution over the various vibrational modes is expected to be highly statistical. From this bath of excited states, dissociation finally occurs. Thus, in the multiphoton absorption process, the vibrational excitation becomes fully randomized and the ion has lost its memory of the vibration that was initially excited.

A more likely explanation is a sequential dissociation process. While still being localized in the trap, a fragment ion having lost an $\mathrm{H}_{2}$ or $\mathrm{C}_{2} \mathrm{H}_{2}$ unit may absorb additional photons and undergo further fragmentation. Since parent and fragment ions have different vibrational spectra, this process is only possible at certain infrared wavelengths and can only take place if absorption bands of parent and fragment ions coincide. In Figure 3 , one clearly observes that in the spectrum recorded on fragment masses having lost more than an acetylenic unit $\left(>\mathrm{C}_{2} \mathrm{H}_{2}\right.$ ), only bands have disappeared as compared to the $\mathrm{H}_{2} /$ $\mathrm{C}_{2} \mathrm{H}_{2}$-loss spectra and no new bands have appeared, suggesting a sequential fragmentation mechanism.

4.2. Acenaphthene Cation, $\mathbf{C}_{\mathbf{1 2}} \mathbf{H}_{10}{ }^{+}$. The acenaphthene cation did not show any sign of carbon loss under the influence of the FEL radiation, in line with the findings of Ekern et al. who used UV radiation. ${ }^{25}$ The infrared spectrum was therefore recorded by determining the ratio on mass channels 152 $\left(\mathrm{C}_{12} \mathrm{H}_{8}{ }^{+}\right)$and $154\left(\mathrm{C}_{12} \mathrm{H}_{10}{ }^{+}\right)$, which are only marginally resolved in our apparatus. Nevertheless, the spectrum as shown in Figure 4 yields clearly discernible infrared absorption bands.

To the best of our knowledge, no infrared spectra of the 


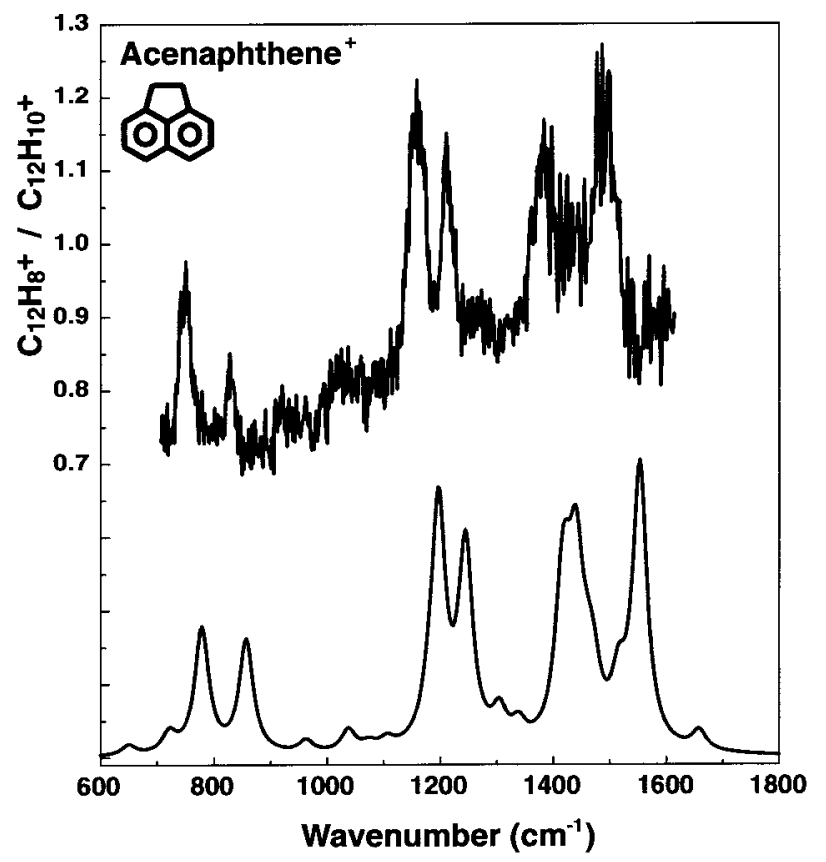

Figure 4. Infrared spectrum of the acenaphthene cation obtained from the ratio between the lighter and heavier sides of the unresolved mass peak (see Figure 2), corresponding roughly to masses of 152 and 154 amu. Good agreement is found with the DFT-calculated spectrum given by the bottom trace.

TABLE 2: Infrared Spectrum of the Acenaphthene Cation

\begin{tabular}{|c|c|c|c|c|c|}
\hline \multicolumn{3}{|c|}{ calculated } & \multicolumn{2}{|c|}{ experimental } & \multirow[b]{2}{*}{ approximate description } \\
\hline$C_{2 v}$ & $\nu_{\text {vib }}$ & $I_{\text {rel }^{a}}$ & $\nu_{\text {vib }}$ & $I_{\text {rel }}$ & \\
\hline$B_{1}$ & 779 & 0.52 & 751 & 0.56 & $\mathrm{CH}$ oop bend/ $\mathrm{CH}_{2}$ wag \\
\hline$B_{1}$ & 857 & 0.46 & 833 & 0.32 & $\mathrm{CH}$ oop bend/ $\mathrm{CH}_{2}$ wag \\
\hline$B_{2}$ & 1197 & 1.00 & 1162 & 1.00 & $\mathrm{CH}_{(2)}$ ip bend \\
\hline$B_{2}$ & 1245 & 0.81 & 1215 & 0.67 & $\mathrm{CH}$ ip bend \\
\hline$B_{2}$ & 1414 & 0.15 & & & CC stretch \\
\hline$A_{1}$ & 1416 & 0.26 & & & CC stretch \\
\hline$B_{2}$ & 1421 & 0.31 & & & asym $\mathrm{CH}_{2}$ scissor \\
\hline$A_{1}$ & 1440 & 0.67 & 1384 & 0.76 & sym $\mathrm{CH}_{2}$ scissor \\
\hline$A_{1}$ & 1459 & 0.16 & & & $\mathrm{CH}_{2}$ scissor/CH ip bend \\
\hline$A_{1}$ & 1471 & 0.22 & & & $\mathrm{CH}$ ip bend/CC stretch \\
\hline$B_{2}$ & 1516 & 0.22 & & & $\mathrm{CC}$ stretch \\
\hline$B_{2}$ & 1551 & 0.20 & & & CC stretch \\
\hline$A_{1}$ & 1553 & 0.97 & 1492 & 0.85 & CC stretch \\
\hline
\end{tabular}

acenaphthene cation have been reported to date. Calculated and experimental absorption frequencies, relative intensities, and approximate descriptions of the vibrational modes and their symmetry in the $C_{2 v}$ point group are listed in Table 2. Calculations were again carried out using the B3LYP method and the D95(d,p) basis set. In Figure 4, the calculated spectrum, convoluted with a $30 \mathrm{~cm}^{-1}$ fwhm Lorentzian line profile, is shown along with the experimental trace. The agreement between calculation and experiment is very good, although a typical blue shift of the calculated frequencies of a few percent is noticed.

The frequencies of out-of-plane $\mathrm{CH}$ bending modes of polyaromatic compounds are known to be determined by the number of adjacent $\mathrm{CH}$ units on an aromatic ring. ${ }^{50}$ The acenaphthene molecule only possesses aromatic rings with three adjacent $\mathrm{CH}$ units, and the out-of-plane $\mathrm{CH}$ bending mode is therefore expected to be located between 750 and $810 \mathrm{~cm}^{-1}$. Thus, at first sight one might assign the band at $751 \mathrm{~cm}^{-1}$ as the aromatic out-of-plane $\mathrm{CH}$ bending mode and, consequently, the band at $833 \mathrm{~cm}^{-1}$ as being due to bending of the aliphatic

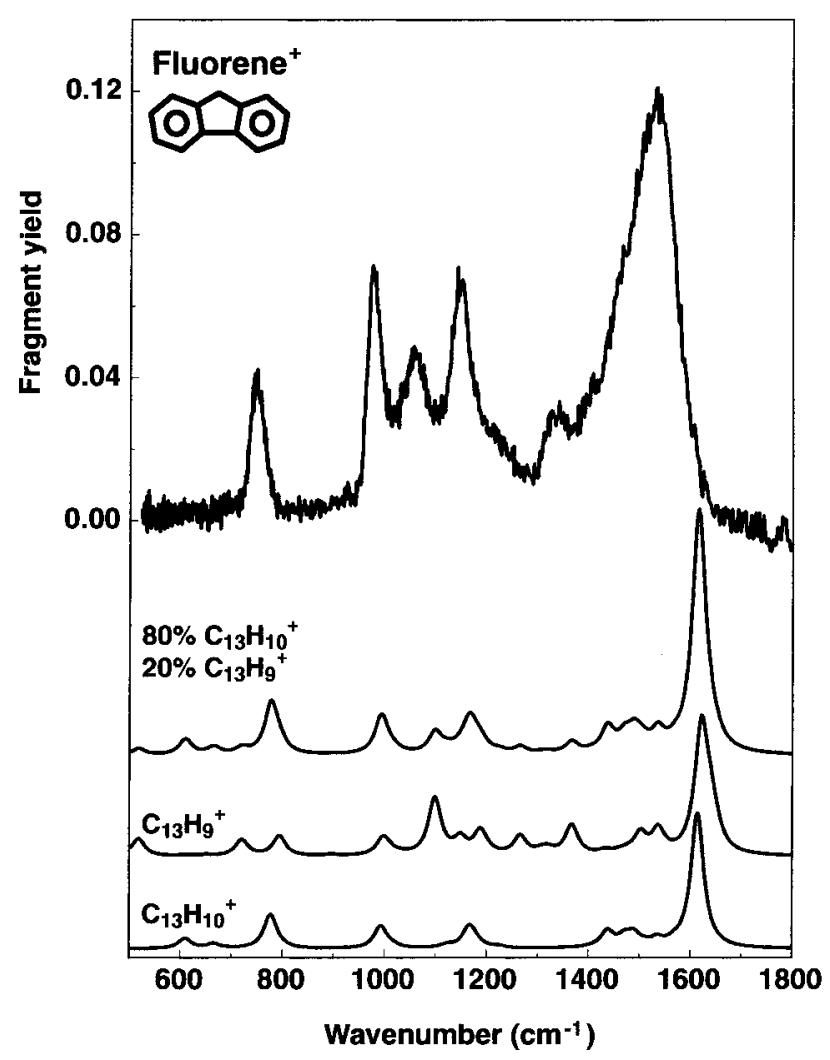

Figure 5. Observed and calculated spectra of cationic fluorene. Calculations were performed for the intact fluorene cation and for the cation which has lost one $\mathrm{H}$ atom from the aliphatic $\mathrm{CH}_{2}$ group during UV ionization. A composite spectrum $\left(20 \% \mathrm{C}_{13} \mathrm{H}_{9}{ }^{+}\right.$and $\left.80 \% \mathrm{C}_{13} \mathrm{H}_{10}{ }^{+}\right)$ is constructed to mimic the observed data.

$\mathrm{CH}_{2}$ groups. However, close inspection of the calculated vibrational displacements reveals that a symmetric and an antisymmetric combination of the aromatic with the aliphatic $\mathrm{CH}$ bending is formed, thus giving rise to the two bands in the spectrum. The two strong features near $1200 \mathrm{~cm}^{-1}$ are found to be due to $\mathrm{CH}$ in-plane bending modes. Though, according to Table 2, the calculations predict that several CC stretching and $\mathrm{CH}_{2}$ scissoring modes are active in the $1400-1600 \mathrm{~cm}^{-1}$ region, only two bands are observed in the experimental spectrum. However, as shown in Figure 4, convolution of the theoretical spectrum with the $30 \mathrm{~cm}^{-1}$ line shape function basically merges all predicted lines into two unresolved features, which is in good agreement with the observed spectrum.

4.3. Fluorene Cation, $\mathrm{C}_{\mathbf{1 3}} \mathrm{H}_{\mathbf{1 0}}{ }^{+}$. Despite the fact that Ekern et al. ${ }^{25}$ reported that cationic fluorene loses only hydrogen under intense irradiation, in this experiment it is possible to observe carbon loss when the FEL is tuned in resonance with an infrared absorption frequency. The spectrum of the fluorene cation as recorded on the $\mathrm{C}_{2} \mathrm{H}_{m}$ loss channel is shown as the top trace of Figure 5, and measured line positions and intensities are listed in Table 3. A spectrum typical of cationic PAHs is observed, i.e., strong and overlapping bands in the $\mathrm{CC}$ stretch region near $1500 \mathrm{~cm}^{-1}$ and weaker but isolated features toward the red end of the spectrum.

Neither experimental (e.g., matrix isolation) data nor theoretical infrared spectra are available for comparison with our spectrum. A B3LYP/D95(d,p) calculation was therefore carried out which yields a $C_{2 v}$-optimized geometry and a vibrational spectrum in which most of the major features can be recognized (see the bottom trace in Figure 5). The band at $746 \mathrm{~cm}^{-1}$ is due to the aromatic $\mathrm{CH}$ out-of-plane bending mode in accordance with its expected position. ${ }^{50}$ The two bands at 977 and 1149 
TABLE 3: Infrared Spectrum of the Fluorene Cation

\begin{tabular}{|c|c|c|c|c|c|}
\hline \multicolumn{3}{|c|}{ calculated } & \multicolumn{2}{|c|}{ experimental } & \multirow[b]{2}{*}{ approximate description } \\
\hline$C_{2 v}$ & $\nu_{\mathrm{vib}}$ & $I_{\mathrm{rel}}{ }^{a}$ & $v_{\text {vib }}$ & $I_{\text {rel }}$ & \\
\hline$B_{2}$ & 610 & 0.07 & & & ring deformation \\
\hline$B_{1}$ & 666 & 0.04 & & & $\mathrm{CH}_{(2)}$ oop bend \\
\hline$B_{1}$ & 778 & 0.25 & 746 & 0.37 & $\mathrm{CH}$ oop bend \\
\hline \multirow[t]{2}{*}{$B_{2}$} & 994 & 0.16 & 977 & 0.65 & asym breathing \\
\hline & & & 1058 & 0.43 & $\mathrm{~b}$ \\
\hline$B_{2}$ & 1167 & 0.14 & 1149 & 0.61 & $\mathrm{CH}_{(2)}$ ip bend \\
\hline \multirow[t]{2}{*}{$A_{1}$} & 1177 & 0.04 & & & $\mathrm{CH}$ ip bend \\
\hline & & & 1212 & 0.21 & \\
\hline$A_{1}$ & 1438 & 0.10 & 1334 & 0.27 & $\mathrm{~b}$ \\
\hline$A_{1}$ & 1471 & 0.07 & 1402 & 0.27 & $\mathrm{CH}_{2}$ scissor/CH ip bend \\
\hline$B_{2}$ & 1490 & 0.09 & 1466 & 0.55 & $\mathrm{CC}$ stretch/CH ip bend \\
\hline$B_{2}$ & 1536 & 0.05 & 1504 & 0.68 & $\mathrm{CC}$ stretch/CH ip bend \\
\hline$B_{2}$ & 1615 & 1.00 & 1532 & 1.00 & $\mathrm{CC}$ stretch/CH ip bend \\
\hline
\end{tabular}

${ }^{a}$ Only bands with an $I_{\text {rel }}$ of $>0.03$ are listed. ${ }^{b}$ Possibly due to the $\mathrm{C}_{13} \mathrm{H}_{9}{ }^{+}$fragment (see the text).

$\mathrm{cm}^{-1}$ are well-reproduced in the calculations and are due to antisymmetric breathing of the two aromatic rings and in-plane $\mathrm{CH}$ bending, respectively. The band observed at $1058 \mathrm{~cm}^{-1}$, however, is clearly missing in the calculations. The strong feature observed near $1500 \mathrm{~cm}^{-1}$ appears to consist of (at least) three strong unresolved bands, whereas the simulation predicts only one very strong peak and some weak features with intensities of $<10 \%$ of that of the main peak. In addition, this peak is blue shifted by $\sim 5 \%$ with respect to the experimental spectrum. The just resolvable band at $1334 \mathrm{~cm}^{-1}$ may be assigned as the $\mathrm{CH}_{2}$ scissoring mode, although the calculations locate this mode more than $100 \mathrm{~cm}^{-1}$ further to the blue. The other unresolvable features between 1400 and $1600 \mathrm{~cm}^{-1}$ are due to various combinations of $\mathrm{CC}$ stretching and $\mathrm{CH}$ in-plane bending vibrations.

As to the question of why the band observed at $1058 \mathrm{~cm}^{-1}$ is missing in the calculations, we note that it is known from high-resolution mass spectrometry (see Figure 41 of ref 25) that the $\mathrm{H}$ atoms of the aliphatic $\mathrm{CH}_{2}$ group are attached rather loosely. ${ }^{51}$ In fact, partial dehydrogenation during UV ionization indeed occurs, judging from the width of the parent ion peak in the TOF spectrum of Figure 2. It could therefore very well be that we also observe the infrared spectrum of the partially dehydrogenated fluorene ion. We therefore calculated the spectrum of $\mathrm{C}_{13} \mathrm{H}_{9}{ }^{+}$, which appears to resemble that of the fluorene cation fairly closely, though a relatively strong (0.46) in-plane $\mathrm{CH}$ bending mode appears at $1099 \mathrm{~cm}^{-1}$, a blue shift of $40 \mathrm{~cm}^{-1}$ or $\sim 3.7 \%$ with respect to the observed band. In addition, the calculation yields a medium-intensity band at 1367 $\mathrm{cm}^{-1}$ which may correspond to the clearly observable shoulder at $1334 \mathrm{~cm}^{-1}$. This assignment appears to be in better agreement with the experimental spectrum than the $\mathrm{CH}_{2}$ scissoring mode of the intact fluorene cation proposed above. In Figure 5, the two calculated spectra have been combined in a $1 / 4 \mathrm{C}_{13} \mathrm{H}_{9}{ }^{+} /$ $\mathrm{C}_{13} \mathrm{H}_{10}{ }^{+}$ratio in an attempt to reproduce the experimental spectrum, and in particular the trident around $1050 \mathrm{~cm}^{-1}$, as closely as possible.

A zero-kinetic energy (ZEKE) photoelectron spectrum of fluorene recorded by Zhang et al. ${ }^{52}$ identifies some of the lowlying vibrations of the fluorene cation. In ZEKE spectroscopy, selection rules are different from those in the infrared spectrum of the ion. Nonetheless, two bands observed in the ZEKE spectrum at 733 and $948 \mathrm{~cm}^{-1}$ may be argued to correspond to the bands observed at 746 and $977 \mathrm{~cm}^{-1}$ in this work, respectively. However, the lines recorded in the cold molecular beam ZEKE spectrum would have to be red-shifted with respect to the room-temperature pure infrared spectrum. Moreover, the

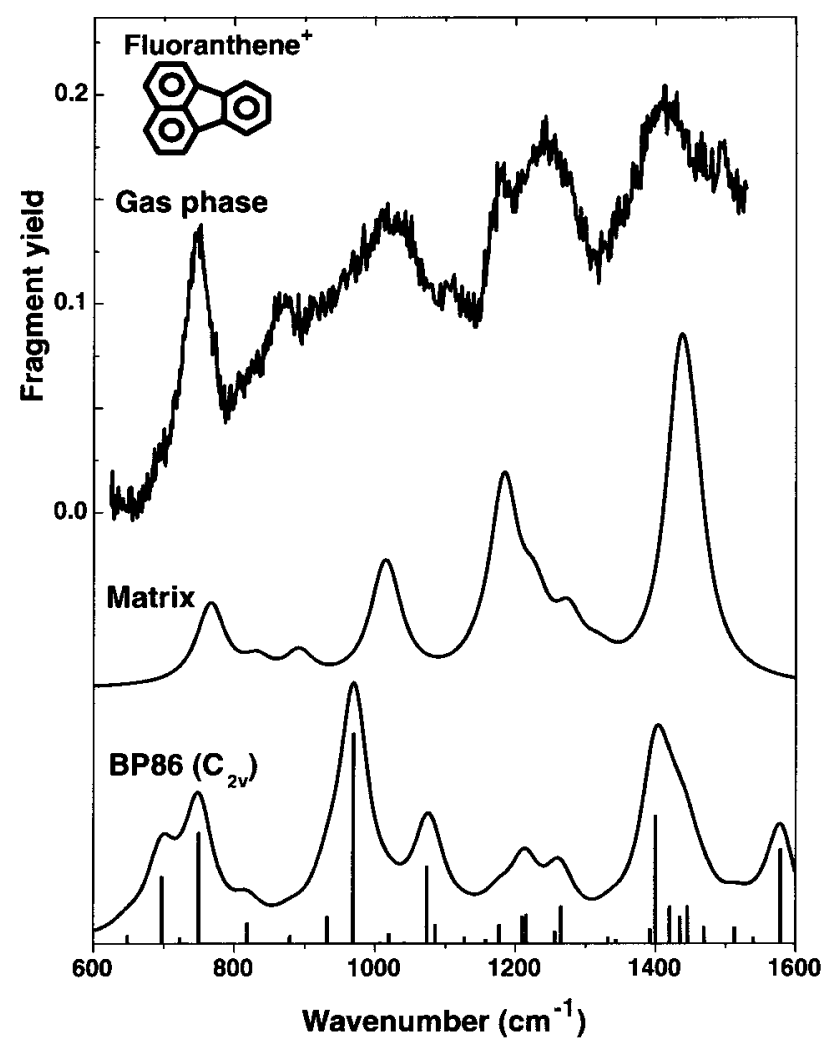

Figure 6. Observed gas phase spectrum for the fluoranthene cation. Although a fairly good match is found with the matrix data of Hudgins et al. ${ }^{24}$ comparison with the theoretical spectrum, calculated in the $C_{2 v}$ geometry using the BP86 functional, yields substantial discrepancies due to electronic state mixing (see the text).

line at $948 \mathrm{~cm}^{-1}$ in the ZEKE spectrum was tentatively assigned as a combination band by Zhang et al., whereas the intensities of the band at $977 \mathrm{~cm}^{-1}$ in our spectrum and at $994 \mathrm{~cm}^{-1}$ in the calculations point to a fundamental band. We therefore conclude that the two spectra are complementary.

4.4. Fluoranthene Cation, $\mathbf{C}_{16} \mathrm{H}_{10}{ }^{+}$. Although infrared absorption strengths of the fluoranthene cation are substantially weaker than for other PAH cations, ${ }^{24}$ it is possible to dissociate this species using the infrared radiation of FELIX. Not only are the absorptions weak, but it also appears particularly difficult to resolve spectral features for this species. Nonetheless, the measurements reproduce very well, giving us a fair confidence in the experimental spectrum shown in Figure 6, which represents an average over four individual wavelength scans.

In contrast to the other species studied in this work, both matrix isolation experimental data ${ }^{24}$ and theoretical calculations 9 are available for the fluoranthene cation. The line positions and intensities extracted from the matrix isolation data were convoluted with a $50 \mathrm{~cm}^{-1}$ Lorentzian line shape function to facilitate easier comparison in Figure 6. Despite the extensive line broadening in our spectrum, there appears to be a fairly good match for most of the main absorption bands. For instance, the feature near $1000 \mathrm{~cm}^{-1}$ appears to consist of two unresolved bands of nearly identical intensity in both spectra (see Table 4). On the other hand, the $\mathrm{CH}$ out-of-plane bending mode due to four adjacent $\mathrm{CH}$ units, which is readily identified at 748 $\mathrm{cm}^{-1}$ in our spectrum, is observed with a 2-3 times lower relative intensity in the matrix. In addition, the intensity distribution in the $1170-1300 \mathrm{~cm}^{-1}$ region shows some discrepancy. It was noted by Hudgins et al. ${ }^{24}$ that the matrixisolated spectrum of this cation suffers particularly from obscuration by neutral bands. 
TABLE 4: Infrared Spectrum of the Fluoranthene Cation

\begin{tabular}{|c|c|c|c|c|c|c|}
\hline \multicolumn{3}{|c|}{ calculated $^{a}$} & \multicolumn{2}{|c|}{ this work } & \multicolumn{2}{|c|}{ matrix $^{b}$} \\
\hline$C_{2 v}$ & $v_{\mathrm{vib}}$ & $I_{\mathrm{rel}^{c}}$ & $v_{\mathrm{vib}}$ & $I_{\text {rel }}$ & $\nu_{\mathrm{vib}}$ & $I_{\text {rel }}$ \\
\hline$B_{2}$ & 697 & 0.32 & & & & \\
\hline$B_{1}$ & 749 & 0.53 & 748 & 0.70 & 766 & 0.29 \\
\hline$B_{1}$ & 819 & 0.10 & & & 832 & 0.07 \\
\hline$B_{2}$ & 932 & 0.13 & 869 & 0.50 & 891 & 0.10 \\
\hline$B_{2}$ & 969 & 1.00 & $1008^{d}$ & 0.45 & $1010^{d}$ & 0.45 \\
\hline & & & $1042^{d}$ & 0.35 & $1020^{d}$ & 0.45 \\
\hline$B_{2}$ & 1074 & 0.37 & & & & \\
\hline$A_{1}$ & 1086 & 0.09 & & & & \\
\hline$A_{1}$ & 1177 & 0.09 & 1110 & 0.35 & 1148 & 0.03 \\
\hline$B_{2}$ & 1210 & 0.13 & 1181 & 0.65 & 1183 & 0.68 \\
\hline$B_{2}$ & 1216 & 0.14 & & & 1226 & 0.22 \\
\hline$A_{1}$ & 1265 & 0.18 & 1242 & 0.85 & 1275 & 0.18 \\
\hline$A_{1}$ & 1400 & 0.61 & 1410 & 1.00 & 1434 & 1.00 \\
\hline$B_{2}$ & 1420 & 0.18 & & & & \\
\hline$A_{1}$ & 1435 & 0.13 & & & & \\
\hline$A_{1}$ & 1446 & 0.18 & & & 1454 & 0.40 \\
\hline$A_{1}$ & 1578 & 0.45 & & & & \\
\hline
\end{tabular}

${ }^{a}$ BP86 density functional (see the text). ${ }^{b}$ From ref $24 .{ }^{c}$ Only bands with an $I_{\text {rel }}$ of $>0.08$ are listed. ${ }^{d}$ Unresolved bands.

It was recently pointed out by Bauschlicher et al. ${ }^{9}$ that a DFT calculation of the fluoranthene cation suffers severely from a low-lying $\left(\approx 1500 \mathrm{~cm}^{-1}\right)$ electronically excited state. The calculation shows that optimization of the geometry using the B3LYP functional results in a distortion away from the $C_{2 v}$ structure. It was also shown that a calculation using the BP86 functional, which retains the $C_{2 v}$ structure, yields better agreement with the experimental matrix-isolated spectrum. In Figure 6, we therefore reproduce the BP86/D95(d,p)-calculated spectrum, which is practically identical to the spectrum of ref 9 calculated in the 4-31G basis. From a comparison of the theoretical spectrum to either of the experimental spectra, it is obvious that the difficulty of the electronic state mixing introduces severe discrepancies for this ion. Therefore, the correspondence between experimental and theoretical absorption bands, as suggested in Table 4, is highly speculative and mode descriptions have been omitted here.

\section{Conclusion}

Infrared multiphoton dissociation spectra are presented for cationic indane, acenaphthene, fluorene, and fluoranthene. Spectra are generally found to be in good agreement with theoretical spectra calculated using the B3LYP density functional, except for the fluoranthene cation. For all species, the strongest bands are found in the in-plane $\mathrm{CH}$ bending and $\mathrm{CC}$ stretching region roughly between 1300 and $1600 \mathrm{~cm}^{-1}$, as is typical for cationic PAHs.

In view of the astrophysical relevance, it is interesting to note the relatively strong bands at $990 \mathrm{~cm}^{-1}$ in the indane spectrum and at $977 \mathrm{~cm}^{-1}$ in the fluorene spectrum. They are located in a region where the UIR spectrum is nearly flat, right between the strong 8.6 and $11.3 \mu \mathrm{m}$ features, which forces one to conclude that these ions are unlikely to give a significant contribution to the interstellar UIR emission. Both bands are due to breathing modes of the aromatic ring(s). In acenaphthene, however, no significant intensity is found in the $10 \mu \mathrm{m}$ region, and inspection of the calculated modes reveals that breathing of the aromatic rings does not occur. If we compare this observation to available matrix and DFT data on small fivemembered species, it appears that species having an aromatic ring attached to the pentagonal ring but not fused to any other aromatic ring possess a significant absorption band near $10 \mu \mathrm{m}$. Examples are indane, fluorene (this work), and benzo[ $a]-$ fluoranthene. ${ }^{9,24}$ On the other hand, in species containing only aromatic rings fused to at least one other aromatic ring, such as acenaphthene (this work) and benzo[k]fluoranthene, ${ }^{9}$ the breathing vibration is quenched. In accordance with this hypothesis, strong absorptions around $10 \mu \mathrm{m}$ are observed in the fluoranthene cation spectrum as well, although the vibrational motion remains unclear due to the poor agreement with theory. Note that in regular PAHs, each aromatic ring is (by definition) attached to at least one other aromatic ring, and therefore, the strong ionic band near $10 \mu \mathrm{m}$ is not observed. Note also that in our spectra of both the indane and fluorene cation, the relative intensity of this breathing mode exceeds its calculated value by a factor of $\sim 4$, and that it is even more intense than the $\mathrm{CH}$ out-of-plane bending mode.

Acknowledgment. We gratefully acknowledge the expert technical support by A. J. A. van Roij and by the FELIX staff. This work is part of the research program of FOM, which is financially supported by the Nederlandse Organisatie voor Wetenschappelijk Onderzoek (NWO).

\section{References and Notes}

(1) Leger, A.; Puget, J. L. Astron. Astrophys. 1984, 137, L5.

(2) Allamandola, L. J.; Tielens, A. G. G. M.; Barker, J. R. Astrophys. J. Lett. 1985, 290, L25.

(3) Allamandola, L. J.; Tielens, A. G. G. M.; Barker, J. R. Astrophys. J. Lett. 1985, 290, L25; Astrophys. J. Suppl. Ser. 1989, 71, 733.

(4) Sloan, G. C.; Hayward, T. L.; Allamandola, L. J.; Bregman, J. D.; DeVito, B.; Hudgins, D. M. Astrophys. J. Lett. 1999, 513, L65.

(5) DeFrees, D. J.; Miller, M. D.; Talbi, D.; Pauzat, F.; Ellinger, Y. Astrophys. J. 1993, 408, 530.

(6) Joblin, C.; Tielens, A. G. G. M.; Geballe, T. R.; Wooden, D. H. Astrophys. J. Lett. 1996, 460, L119.

(7) Allamandola, L. J.; Hudgins, D. M.; Sandford, S. A. Astrophys. J. Lett. 1999, 511, L115.

(8) Langhoff, S. R. J. Phys. Chem. 1996, 100, 2819.

(9) Bauschlicher, C. W., Jr.; Hudgins, D. M.; Allamandola, L. J. Theor. Chem. Acc. 1999, 103, 154.

(10) Szczepanski, J.; Roser, D.; Personette, W.; Eyring, M.; Pellow, R.; Vala, M. J. Phys. Chem. 1992, 96, 7876.

(11) Hudgins, D. M.; Sandford, S. A.; Allamandola, L. J. J. Phys. Chem. 1994, 98, 4243 .

(12) Joblin, C.; d'Hendecourt, L.; Léger, A.; Défourneau, D. Astron. Astrophys. 1994, 281, 923.

(13) Cherchneff, I.; Barker, J. R. Astrophys. J. Lett. 1989, 341, L21.

(14) Shan, J.; Suto, M.; Lee, L. C. Astrophys. J. 1991, 383, 459.

(15) Brenner, J. D.; Barker, J. R. Astrophys. J. Lett. 1992, 388, L39.

(16) Schlemmer, S.; Cook, D. J.; Harrison, J. A.; Wurfel, B.; Chapman, W.; Saykally, R. J. Science 1994, 265, 1686.

(17) Cook, D. J.; Schlemmer, S.; Balucani, N.; Wagner, D. R.; Steiner, B.; Saykally, R. J. Nature 1996, 380, 227.

(18) Cook, D. J.; Schlemmer, S.; Balucani, N.; Wagner, D. R.; Harrison, J. A.; Steiner, B.; Saykally, R. J. J. Phys. Chem. A 1998, 102, 1465.

(19) Piest, J. A.; von Helden, G.; Meijer, G. Astrophys. J. Lett. 1999 $520, \mathrm{~L} 75$.

(20) Satink, R. G.; Piest, J. A.; von Helden, G.; Meijer, G. J. Chem. Phys. 1999, 111, 10750.

(21) Oomens, J.; van Roij, A. J. A.; Meijer, G.; von Helden, G. Astrophys. J. 2000, 542, 404.

(22) Piest, J. A.; Oomens, J.; Bakker, J. M.; von Helden, G.; Meijer, G. Spectrosc. Chim. Acta A 2001, 57, 717.

(23) Hudgins, D. M.; Sandford, S. A. J. Phys. Chem. A 1998, 102, 353.

(24) Hudgins, D. M.; Bauschlicher, C. W., Jr.; Allamandola, L. J.; Fetzer, J. C. J. Phys. Chem. A 2000, 104, 3655.

(25) Ekern, S. P.; Marshall, A. G.; Szczepanski, J.; Vala, M. J. Phys. Chem. A 1998, 102, 3498.

(26) Peiris, D. M.; Cheeseman, M. A.; Ramanathan, R.; Eyler, J. R. $J$. Phys. Chem. 1993, 97, 7839.

(27) Stephenson, J. L., Jr.; Booth, M. M.; Shalosky, J. A.; Eyler, J. R.; Yost, R. A. J. Am. Soc. Mass Spectrom. 1994, 5, 886.

(28) Paul, W. Rev. Mod. Phys. 1990, 62, 531.

(29) Oepts, D.; van der Meer, A. F. G.; van Amersfoort, P. W. Infrared Phys. Technol. 1995, 36, 297.

(30) Black, J. G.; Yablonovitch, E.; Bloembergen, N.; Mukamel, S. Phys. Rev. Lett. 1977, 38, 1131. 
(31) Grant, E. R.; Schulz, P. A.; Sudbo, Aa. S.; Shen, Y. R.; Lee, Y. T. Phys. Rev. Lett. 1978, 40, 115.

(32) Bagratashvili, V. N.; Letokhov, V. S.; Makarov, A. A.; Ryabov, E. A. Multiple Photon Infrared Laser Photophysics and Photochemistry; Harwood Academic Publishers: Chur, Switzerland, 1985.

(33) Felker, P. M.; Zewail, A. H. J. Chem. Phys. 1985, 82, 2975.

(34) Callegari, A.; Merker, U.; Engels, P.; Srivastava, H. K.; Lehmann,

K. K.; Scoles, G. J. Chem. Phys. 2000, 113, 10583.

(35) Kerstel, E. R. Th.; Lehmann, K. K.; Mentel, T. F.; Pate, B. H.; Scoles, G. J. Phys. Chem. 1991, 95, 8282.

(36) Stuchebrukhov, A. A.; Marcus, R. A. J. Chem. Phys. 1993, 98, 6044.

(37) Lehmann, K. K.; Scoles, G.; Pate, B. H. Annu. Rev. Phys. Chem. 1994, 45, 241.

(38) McIllroy, A.; Nesbitt, D. J.; Kerstel, E. R. Th.; Pate, B. H.; Lehmann, K. K.; Scoles, G. J. Chem. Phys. 1994, 100, 2596.

(39) Chirokolava, A.; Perry, D. S.; Boyarkin, O. V.; Schmid, M.; Rizzo,

T. R. J. Chem. Phys. 2000, 113, 10068.

(40) Bixon, M.; Jortner, J. J. Chem. Phys. 1968, 48, 715.
(41) Bethardy, G. A.; Perry, D. S. J. Chem. Phys. 1993, 98, 6651.

(42) Bethardy, G. A.; Perry, D. S. J. Chem. Phys. 1993, 99, 9400.

(43) Alimpiev, S. S.; Sartakov, B. G. Laser Chem. 1992, 12, 147.

(44) Stein, S. E.; Rabinovitch, B. S. J. Chem. Phys. 1973, 58, 2438.

(45) Schutte, W. A.; Tielens, A. G. G. M.; Allamandola, L. J. Astrophys. J. 1993, 415, 397 .

(46) Becke, A. D. J. Chem. Phys. 1993, 98, 5648.

(47) Dunning, T. H., Jr.; Hay, P. J. in Modern Theoretical Chemistry; Schäfer, H. S., III, Ed.; Plenum: New York, 1976.

(48) Frisch, M. J., et al. Gaussian 98, revision A.7; Gaussian, Inc.: Pittsburgh, PA, 1998.

(49) Joblin, C.; Boissel, P.; Léger, A.; d'Hendecourt, L.; Défourneau, D. Astron. Astrophys. 2000, 299, 835.

(50) Hudgins, D. M.; Allamandola, L. J. Astrophys. J. Lett. 1999, 516, L41

(51) Jochims, H. W.; Baumgärtel, H.; Leach, S. Astrophys. J. 1999, 512, 500 .

(52) Zhang, X.; Pitts, D.; Nadarajah, R.; Knee, J. L. J. Chem. Phys. 1997, 107, 8239 . 